\title{
Game Theoretical Interactions of Moving Agents
}

\author{
Wenjian Yu and Dirk Helbing \\ ETH Zurich, UNO D11, Universitätstr. 41, 8092 Zurich, Switzerland
}

\begin{abstract}
Game theory has been one of the most successful quantitative concepts to describe social interactions, their strategical aspects, and outcomes. Among the payoff matrix quantifying the result of a social interaction, the interaction conditions have been varied, such as the number of repeated interactions, the number of interaction partners, the possibility to punish defective behavior etc. While an extension to spatial interactions has been considered early on such as in the "game of life", recent studies have focussed on effects of the structure of social interaction networks.

However, the possibility of individuals to move and, thereby, evade areas with a high level of defection, and to seek areas with a high level of cooperation, has not been fully explored so far. This contribution presents a model combining game theoretical interactions with successdriven motion in space, and studies the consequences that this may have for the degree of cooperation and the spatio-temporal dynamics in the population. It is demonstrated that the combination of game theoretical interactions with motion gives rise to many self-organized behavioral patterns on an aggregate level, which can explain a variety of empirically observed social behaviors.
\end{abstract}

\section{Introduction}

Macroscopic outcomes in a social system resulting from interactions between individuals can be quite different from anyone's intent. For instance, empirical investigations [1] have shown that most colored people prefer multi-racial neighborhoods, and many white people find a certain fraction of other races in their neighborhood acceptable. So one could think that integrated neighborhoods should be widely observed, but empirically this is not true. One rather finds segregated neighborhoods, i.e. separate urban quarters, which also applies to people with different social and economic backgrounds.

This problem is scientifically addressed by mainly two streams of segregation theory [2]: the urban ecological "social distance" tradition in sociology [34] and the "individual preferences" tradition in economics [5]6. The main idea of "social distance" theory is that the differences in culture and interests between social groups are reflected by a separation of their residential areas. 
Yet, the role of social distance and individual preferences is questioned by studies of the American urban housing market, which suggest that racial discrimination and prejudices are the primary factors of residential segregation and concentration of poverty [7. There are three stages in a housing market transaction: first, information about available housing units, second, terms and conditions of sales and financing assistance, and third, the access to units other than the advertised unit [8]. In each stage, the housing agent may behave in a discriminatory way, e.g. withhold information from customers and discourage them. Therefore, the access of minority customers to housing is severely constrained, while the theory of preference-based dynamics assumes that people can relocate freely according to their own preferences, which fails to reflect the real relocation dynamics.

Recent studies [29] point out that the effect of discrimination in racial residential segregation was important in the past, but nowadays, the nature and magnitude of housing discrimination has changed. Minority households who seek to move into integrated or predominantly white areas usually will be able to do so. But the integration of ethnic groups is not widely observed and quite unstable. This is partly because, when people move to a neighborhood, where they constitute an ethnic minority, the previous inhabitants may choose to leave, while some people may be reluctant to enter integrated neighborhoods in which minorities are increasing in number, e.g. because of a decrease in the housing prices. Such migration dynamics, based on seeking preferred neighborhoods, does not necessarily presuppose the discrimination of other people, as we will shortly see.

Assuming that individuals have just a slight preference for neighborhoods, in which the same ethnic background prevails, Schelling has reproduced residential segregation by a simple model [56. Imagine that two groups of people are distributed over a one-dimensional lattice, and assume that everyone defines his/her relevant neighborhood by the four nearest neighbors on either side of him/her. Moreover, assume that each individual prefers at least four of his/her eight nearest neighbors to belong to same ethnic group as he/she does. Considering himself/herself, this implies a small majority of five out nine people in the considered neighborhood. If the condition is not met, he or she is assumed to move to the nearest site/cell that satisfies his or her desire. Hence, the model does not assume optimizing location choice, just satisficing behavior. Nevertheless, it produces segregation patterns. 


\subsection{Migration, Game Theory, and Cooperation}

In game theoretical terms, this movement to a more favorable neighborhood could be reflected by a higher payoff to persons who moved, called "migrants" in the following. Migratory behavior aiming at higher payoffs is called "success-driven motion" [10,11,12. As we will show in later sections, success-driven motion can reproduce residential segregation and some other observed phenomena of population dynamics as well, like population succession (i.e. cycles of changing habitation) [13. We will also study how a change of the spatial population structure can affect the level and evolution of cooperation in a population.

It could be thought that natural selection, implying competition between individuals, would result in selfish rather than cooperative behavior [14]. Nevertheless, cooperation is widely observed also in competitive settings, from bacteria, over animals to humans [15]16]. Game theory [17,18 19 20 has been regarded as a powerful framework to investigate this problem, as it can be used to quantify the interactions between individuals.

The mathematical description of tendencially selfish behavior is often based on the prisoner's dilemma game, in which the "reward" $R$ represents the payoff for mutual cooperation, while the payoff for defection (cheating) on both sides is reflected by the "punishment" $P$. Unilateral cooperation will incur the so-called "sucker's payoff" $S$, while the defector gains T, the "temptation". Given the inequalities $T>R>P>S$ and $2 R>T+S$, defection is tempting and cooperation is risky, so that defection is expected to be dominating, although mutual cooperation improves the average payoff.

In a well-mixed population, where an individual interacts with all the others, a defector's payoff is always higher than the average payoff, which leads to a prosperity of defectors. In an evolutionary setting as it is described by the replicator dynamics [21, only those strategies that generate above-average payoffs have the chance to spread. Therefore, from an evolutionary perspective, there will be no cooperators in the end, which contradicts the observed cooperation in reality.

A variety of mechanisms has been proposed to explain the considerable level of cooperation observed under certain circumstances. These include kin selection, direct reciprocity, indirect reciprocity, group selection and network reciprocity 14. In particular, it is interesting that the spatial population structure can significantly change the level of cooperation in a population [22 23]. While there could be a co-evolution of social structure 
and cooperation due to migratory behavior, this subject has not been well studied in the past.

\subsection{Migration and Population Succession}

Population succession plays an important role in the change of the population structure in urban areas. The term succession is viewed by ecologists as an orderly sequence of changes in the composition or structure of a biotic community [24]. Later on, it was also used to describe the change in neighborhood and urban land use [25. For example, Cressey [13] analyzed the movements of immigrants in Chicago from 1898 to 1930 by census tracts and provided a detailed picture of the succession processes of specific cultural groups. Interestingly, these processes show a cycle of invasion, conflict, recession, and reorganization [26].

The "invasion" can be initiated by a few "pioneers", who manage to enter a new area dominated by "older" residents. For example, some rich colored people may move to a white-only neighborhood, where the housing price is relatively high. In this stage, discrimination by a housing agency could prevent the integration of cultural groups by constraining information to minority groups [8]. But nowadays, housing discrimination is illegal in many countries, and the hostility toward minority group members has declined. Therefore, the possibility of successful entrance for those "pioneers" is much larger.

Following the initial "invaders", invasions can happen on a much larger scale, in particular if housing prices go down. Then, previous residents, who want to sell their houses at a good price, are eventually replaced. People may even move without reasons, just following the trend of what the others do, which is a kind of collective herding behavior [27]. Accompanying this replacement process, the changing composition of the population may cause conflicts due to incompatible cultural values and prejudices [28].

Eventually, the population composition is turned over. Although the new group can dominate the residential area, it takes time to reorganize the environment such as churches and to create it according to their own requirements. The cycle can repeat as the previous "invaders" become established residents. Therefore, there is a typical life circle of a neighborhood [25], which involves five stages: development, transition, downgrading, thinning out, and renewal. If the facilities in a neighborhood are running down, residents start to seek new places and abandon the previous one. In this case, there is usually little conflict. In the sub- 
sequent renewal stage, new people move in at low prices. By renovating the houses, the quarter eventually revives.

\subsection{Co-evoultion of Social Structure and Cooperation}

In order to model such intentional movement of individuals, spatial effects must be explicitly considered. Spatial games based on lattices [22] would allow one to study such effects. In conventional spatial games, individuals are uniformly distributed in the simulation area, and change or repeat their strategies, following a certain updating rule. For instance, an individual is assumed to unconditionally adopt the most successful strategy within the neighborhood. On the one hand, this creates typical spatio-temporal pattern. On the other hand, spatial structures play an important role in the maintenance of cooperation. In the iterative prisoner's dilemma, for example, clusters of cooperators are beneficial to cooperators [12, while the evolving spatial structure in the snowdrift game [23], may inhibit cooperation. Conventional spatial games, however, neglect the possibility of individuals to move or migrate, although mobility is a well-known fact of daily social interactions. We will see that the movement of individuals, e.g. population succession, can change the spatial structure of a population significantly, and influence the evolution of cooperation dramatically.

The above mentioned social processes that are related to the migratory behavior of people, can be well integrated into the framework of spatial games. Focusing on success-driven motion, we will in the following, study the combination of migration, strategic interactions and learning (specifically imitation). Numerical simulations show that success-driven motion is a mechanism that can promote cooperation via self-organized social structures. Moreover, very surprisingly, a certain degree of fluctuations ("noise") can even enhance the level of cooperation.

\section{Spatial Games with Mobility}

\subsection{Classification}

Spatial games with mobility ("mobility games") could be classified as follows:

1. Mobility may take place in physical (geographic) space with one, two or three dimensions, or it may occur in abstract space (e.g. opinion space). Rather than a regular (grid) space, one may also have a (fixed or dynamically changing) network structure (e.g. friendship network). 
2. One may distinguish between games with continuous and with discrete motion. The first ones may be considered as particular cases of differential games [29] and shall be called "motion games". The second ones will be named "migration games" and can be implemented, for example, in terms of cellular automata (particularly, if a parallel update is performed).

3. The mobility game may be deterministic or "noisy", i.e. influenced by fluctuations. Such "noise" may be introduced by stochastic update rules (e.g. a random sequential update or the Fermi rule [23].

4. Multiple occupation of a certain location may be possible, allowing for the agglomeration of individuals, or it may be prohibited. In the latter case, spatial exclusion requires spatial capacity constraints and/or the respect or protection of some "private territory".

5. In mobility games with spatial exclusion, empty sites are needed. Therefore, the density of free locations ("sites") is a relevant model parameter.

6. The frequency, rate, or speed of mobility may be relevant as well and should be compared with other time scales, such as the frequency of strategy changes, or the average lifetime of a spatial cluster of individuals. Depending on the specification of the game, after a transient time one may find everything from chaotic patterns [22] upto frozen patterns [30,31]. This may be expressed by a viscosity parameter [32].

7. Mobility may be random or directed (e.g. "success-driven"). Directed mobility may depend on the expected payoff in a certain time point (iteration), or it may depend on the cumulative payoff (i.e. the accumulation of wealth by individuals).

8. If age, birth and death are considered, one speaks of "demographic games" 33.

In the following, we will primarily focus on games with success-driven migration in two-dimensional geographic space. Furthermore, we will assume simple strategies (such as all-cooperate or all-defect), no memory and no forecasting abilities.

\subsection{Individual Decision Making and Migration}

In "migration games", each individual $I$ is located at the position $\boldsymbol{X}_{\boldsymbol{I}}$ of a discrete grid and applies a certain strategy $i=i(I, \boldsymbol{X}, t)$ when interacting with individuals $J$ at locations $\boldsymbol{X}_{\boldsymbol{J}}$ in the neighborhood $\mathcal{N}=\mathcal{N}\left(\boldsymbol{X}_{\boldsymbol{I}}\right)$, who apply strategies $j=j\left(J, \boldsymbol{X}^{\prime}, t\right)$ (see Fig. 1). The interactions at time $t$ are quantified by the payoffs $P_{i j}$. The overall payoff for individual $I$ resulting 
from interactions with all individuals $J$ in the neighborhood $\mathcal{N}\left(\boldsymbol{X}_{\boldsymbol{I}}\right)$ at time $t$ is

$$
P_{I}(t)=P_{I}\left(\boldsymbol{X}_{\boldsymbol{I}}, t\right)=\sum_{J: \boldsymbol{X}_{\boldsymbol{J}} \in \mathcal{N}\left(\boldsymbol{X}_{\boldsymbol{I}}\right)} P_{i j}(J)
$$

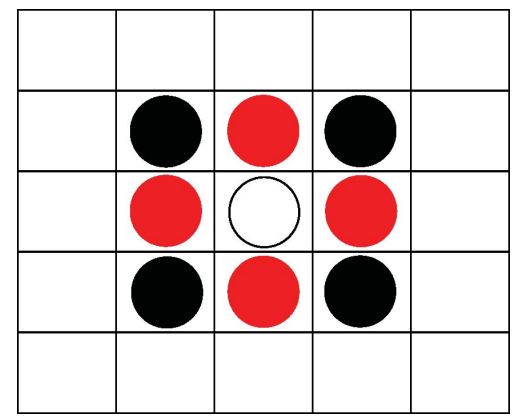

Fig. 1. The focal individual $I$ is represented by the empty circle. It can, for example, interact with the $k=4$ nearest individuals (red) or with $k=8$ neighbors, which includes four next-nearest neighbors (black).

We assume that all individuals prefer places that can provide higher payoff. However, their movements are restricted by their mobilities and the number of free locations, as one can only move to empty places. In our model, the mobility is reflected by the migration range $M$, which could be assumed constant (see Fig. 22 or as a function of cumulative payoffs

$$
C_{I}(t)=\sum_{t^{\prime}=0}^{t}\left[P_{I}\left(t^{\prime}\right)-c_{I}\left(t^{\prime}\right)\right] .
$$

Here, the cost of each movement $c_{I}(t)$ may be specified proportionally to the distance $d_{I}(t) \leq M$ moved:

$$
c_{I}(t)=\beta d_{I}(t) .
$$

$\beta$ is a constant.

When the migration range $M$ is restricted by the cumulative payoff, one may set

$$
M(t)=\left\lfloor\alpha C_{I}(t)\right\rfloor,
$$


where \lfloor\rfloor rounds down to the next integer $\leq \alpha C_{I}(t)$ and $\alpha$ is a constant, which is set to $\frac{1}{\beta}$ in the following.
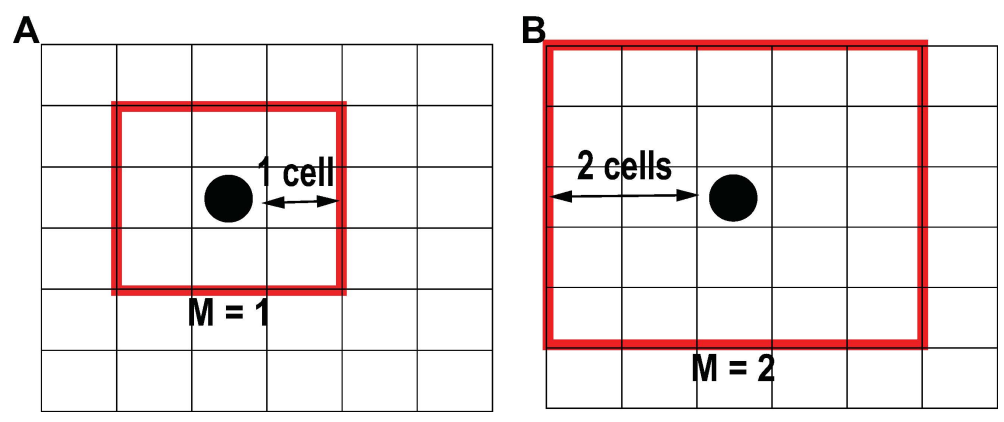

Fig. 2. (A) When $M=1$, the focal individual (black circle) can migrate within the red square (Moore neighborhood of range 1). (B) Here, the migration neighborhood is chosen as Moore neighborhood of range $M=$ 2 .

The expected payoff for individual $I$ applying strategy $i$ at a free location $\boldsymbol{X}^{\prime}$ within the migration range can be calculated as

$$
P_{I}\left(\boldsymbol{X}^{\prime}, t\right)=\sum_{J^{\prime}: \boldsymbol{X}_{J^{\prime}} \in \mathcal{N}\left(\boldsymbol{X}^{\prime}\right)} P_{i j^{\prime}}\left(J^{\prime}\right)
$$

where $j^{\prime}=j^{\prime}\left(J^{\prime}, \boldsymbol{X}^{\prime}, t\right)$ are the strategies of the individuals $J^{\prime}$ located within the neighborhood $\mathcal{N}^{\prime}=\mathcal{N}\left(\boldsymbol{X}^{\prime}\right)$ centered at $\boldsymbol{X}^{\prime}$. Here, we assume that an individual can determine this value by testing a neighborhood $\mathcal{N}^{\prime}$ by means of some kind of "fictitious play" with its residents.

All individuals are assumed to maximize the expected payoff by migrating to the place that promises the highest payoff. Consequently, the payoff at the new destination for $I$ is expected to be

$$
P_{I}^{e}(t+1)=\max _{\boldsymbol{X}^{\prime} \in \mathcal{A}} P_{I}\left(\boldsymbol{X}^{\prime}, t\right),
$$

where $\mathcal{A}$ represents the area within the migration range $M$ around $\boldsymbol{X}_{\boldsymbol{I}}$.

In our model, individuals decide to move, if the expected payoff at the new position $P_{I}^{e}(t+1)$ satisfies the short-term cost-benefit condition

$$
P_{I}^{e}(t+1)-P_{I}(t)>c_{I}(t)
$$


If we assume that the cost of each movement is small, and the new neighborhood yields a comparatively high cumulative payoff over the time period the individual stays at that place, then the cost of movement can be neglected.

Finally, if two or more places promise the same maximum payoff, we assume that an individual will choose the closest one. If both, the payoff and distance are the same, then the individual will randomly choose one of the closest locations in our simulation. Since all the other individuals also seek new places that can increase their payoffs, neighborhoods may change quickly, and the resulting payoff may fall below the expectations before the move (during the fictitious play step).

\subsection{Learning}

Learning allows individuals to adapt their behaviors in response to other people's behaviors in an interactive decision making setting. Basic learning mechanisms are, for example, unconditional imitation, best reply, and reinforcement learning [34].

Unconditional imitation means that people copy the behaviors of others. For instance, we can assume that, when $i$ reaches a new place, it imitates the most successful strategy within the neighborhood (if it is more successful than the own strategy), or the most frequently used strategy in past interactions. It should be underlined, that it is not easy to identify the future strategy of another individual from its displayed past behavior. However, in the simplified context of a prisoner's dilemma, where individuals are assumed to play either all-defect or all-cooperate, one's behavior reveals the strategy directly. It may nevertheless change before the next iteration due to learning of the neighbors.

Reinforcement learning is a kind of backward-looking learning behavior, i.e. people tend to take the actions that yielded high payoffs in the past. Assuming that an individual has a set of strategies, among which he or she chooses with a certain probability, this probability is increased, if the gained payoff exceeds a certain threshold value ("aspiration level") [35 36].

Best reply is a sophisticated learning model as well. According to it, people choose their strategies based on the expectation of what the others will do in a way that maximizes their expected payoff. The ability to forecast depends on one's belief about the behaviors of the others. For instance, one can try to determine the distribution of the neighbors' previous actions, and select the own strategy, which replies to it in the best way. 
For simplicity, players in our model only interact with the $k=4$ nearest neighbors and adapt their strategies by unconditional imitation. Assuming that the updating rules of all the individuals are the same, we can specify a simple migration game as follows:

1. An individual moves to a new position that is located inside the migration range $M$ and maximizes the expected payoff.

2. The individual imitates the most successful strategy within the interaction neighborhood $\mathcal{N}$, if it is more successful than the own strategy.

Note that changing the sequence leads to a different dynamics, and the implementation of migration and learning can be varied in many ways. Therefore, spatial games with mobility promise to be a rich research field. In the following, we will restrict ourselves to some of the simplest specifications.

The above mentioned framework of spatial games with migration specifies a kind of cellular automata (CA) model, in which space and time are discrete. Furthermore, the set of actions performed in CA models usually depends on the last time step only, which allows for high-performance computing of large-scale scenarios. CA models have been successfully applied to describe a variety of social and physical systems [33 37 38]. The outcomes of social and physical interactions can be very well integrated into them, which can create many interesting dynamics, like the "game of life" 39].

\section{Simulation Results and Discussion}

In the following, we perform a random sequential update of the individual (i.e. their migration and learning steps), which is to be distinguished from the parallel update assumed in [2230]. For a discussion of some related advantages, see [40. Our numerical simulations are performed on $49 \times 49$ grids with $40 \%$ empty sites, i.e. a density of 0.6 and periodic boundary conditions.49x49 grids were chosen for better visibility, while the statistical evaluations (see Figs. 6 and 7) were done with $99 \times 99$ grids for comparability with Nowak's and May's spatial games [22]. In the prisoner's dilemma, the color code is chosen as follows: blue $=$ cooperator, red $=$ defector, green $=$ defector who became a cooperator, yellow $=$ cooperator who turned into a defector in the last iteration. In other games, blue $=$ player of group 1 , red $=$ player of group 2 , green $=$ player of group 2 who became a player of group 1 , yellow $=$ player of group 1 who turned into a player of group 2. White always corresponds to an empty site. 


\subsection{Spontaneous Pattern Formation and Population Structure}

In the migration game, individual preferences are reflected by the payoff matrix, which quantifies the possible outcomes of social interactions. Fig. 3 shows a variety of patterns formed when the payoff matrix is modified. The outcomes from interactions between individuals are the driving forces changing the population structure in space. In the following, we will call all the individuals, who apply the same strategy, a "group" $g$. Group 1 is represented in blue, group 2 in red. The size $N_{g}$ of group $g$ is constant in true only in the migration-only case without learning. Otherwise, strategy changes imply changes of individuals between groups and changes in group size. We will distinguish the three social relations between groups: (i) both groups (or, more exactly speaking, their individuals) like each other $\left(P_{12}=P_{21}=1\right)$, (ii) group 1 is neutral with respect to group 2 , but group 2 dislikes group $1\left(P_{12}=0, P_{21}=-1\right)$, and (iii) intra-group interactions are more favored than inter-group interactions $\left(P_{11}=P_{22}=1\right.$, $\left.P_{12}=P_{21}=0.5\right)$. Intra-group affiliation is always preferred or at least neutral $\left(P_{11}=P_{22}=1\right.$ or $\left.P_{11}=P_{22}=0\right)$. As people are allowed to move, when they like each other, a strong integration of populations can be observed. However, if the affinity is unilateral, then one population tends to evade the invasion of the other. Assuming the same mobility in both populations, in the current setting of our simulations the chasing population gains the majority in the end, if unconditional imitation is considered.

Residential segregation emerges through the interactions of individuals, not only when they dislike each other, but also if individuals within the same group like each other more than individual from other groups. When inter-group interactions result in smaller payoffs, people attempt to maximize their payoff by agglomerating with the same kind of people, which eventually results in the segregation of different groups as a side effect.

Some theoretical analysis can be useful to understand the micro-macro link. Let $n_{g 1}$ and $n_{g 2}$ be the average number of individuals of group 1 and 2 , respectively, in the interaction neighborhood of an individual using strategy $i$, i.e. belonging to group $g=i$. Then, the payoff of an individual belonging to group $g \in\{1,2\}$ is

$$
P_{g}=n_{g 1} P_{g 1}+n_{g 2} P_{g 2},
$$

where $P_{g i}$ is the payoff of an individual of group $g$ when meeting an individual using strategy $i$. 


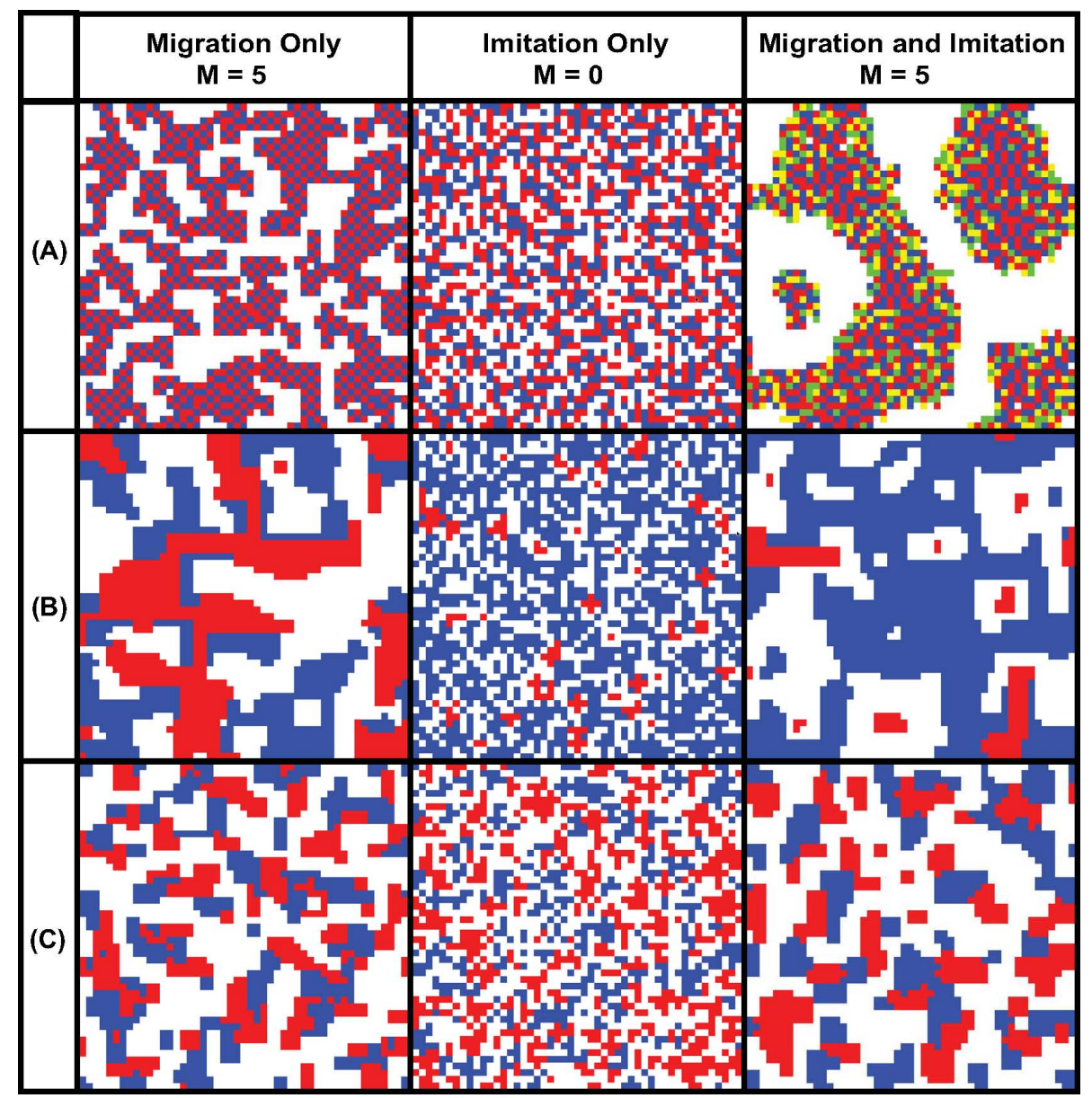

Fig. 3. Simulation results for $49 \times 49$ grids with a density of 0.6 for the migration-only case (left), the imitation-only case of conventional spatial games (middle) and the combination of imitation with migration (right). The color code is chosen as folows: blue $=$ player of group 1 , red $=$ player of group 2, yellow $=$ player of group 1 who turned into a player of group 2 in the last iteration, green $=$ player of group 2 who turned into a player of group 1 in the last iteration, white $=$ empty site. (A) $P_{11}=P_{22}=$ $0, P_{12}=P_{21}=1$. Inter-group interaction is encouraged, which causes the integration of two populations. The combination of migration and imitation results in the co-existence of both groups and the formation of clusters. (B) $P_{11}=P_{22}=P_{12}=1, P_{21}=-1$. When affinity is unilateral, one population keeps approaching the other one, which tends to evade. The combination of migration and imitation leads to the spreading of the chasing group. Co-existence is not observed. (C) $P_{11}=P_{22}=1$, $P_{21}=P_{21}=0.5$. When interactions between groups are less profitable than in the same group, residential segregation occurs as well, but in contrast to (B), it stabilizes. 
The total payoffs for individuals of group $g$ is $N_{g} P_{g}$, while the total payoff of both groups is

$$
T^{\prime}=N_{1} P_{1}+N_{2} P_{2},
$$

where $N_{g}$ is the number of individuals of group $g$, i.e. pursuing strategy $g=i$.

According to success-driven migration, the change of an individual's payoff in the noiseless case is always positive, i.e.

$$
\Delta P_{g}=\Delta n_{g 1} P_{g 1}+\Delta n_{g 2} P_{g 2}>0
$$

with $\Delta n_{g 1}=n_{g 1}(t+1)-n_{g 1}(t)$ and $\Delta n_{g 2}=n_{g 2}(t+1)-n_{g 2}(t)$.

Assuming $P_{12} \gg P_{11}$ and $P_{12}>0$, we will usually have $\Delta P_{1} \approx$ $\Delta n_{12} P_{12}>0$ and $\Delta n_{12}>0$, which leads to a monotonous increase in the number of neighbors of individuals of group 1 . Giving the inequality $P_{11} \gg P_{12}$ and $P_{11}>0$, we analogously obtain $\Delta P_{1} \approx \Delta n_{11} P_{11}>0$ and $\Delta n_{11}>0$. Therefore, when intra-group interactions of group 1 are much stronger than inter-group interactions, and positive, clusters of group 1 will expand, as each migration step will increase the average number of neighbors within group 1 . However, when $P_{11}<0$, the formation of clusters of individuals belonging to group 1 by intra-group interactions is unlikely. When $P_{12}<0$, an individual of group 1 tends to evade members of group 2, which can be regarded as a repulsive effect attempting to keep a certain distance between individuals of different groups [41.

The total change of payoff is given by

$$
\Delta T^{\prime}=N_{1}\left(\Delta n_{11} P_{11}+\Delta n_{12} P_{12}\right)+N_{2}\left(\Delta n_{21} P_{21}+\Delta n_{22} P_{22}\right)>0,
$$

as long as success-driven migration is applied by all the individuals, and changes $\Delta N_{1}$ and $\Delta N_{2}$ in the group sizes are negligible.

Let us now consider a simple example, where $P_{11}=P_{22}=0$. Then, we have

$$
\Delta T^{\prime}=N_{1} \Delta n_{12} P_{12}+N_{2} \Delta n_{21} P_{21}
$$

which reflects the combined effects of interactions between members of group 1 and 2. Furthermore, if $\Delta T^{\prime}>0$, we expect that the number of neighborships between individuals of both groups will monotonously increase. The corresponding macroscopic phenomenon is the spatial integration (mixture) of both groups. However, if $\left(P_{12}+P_{21}\right)<0$, the reduction of inter-group links is likely and will result in residential segregation (see Fig. 3). 
Here we have examined how individual preferences can change the spatial population structure in a very simple social system considering migratory behavior. The revealed social process can, to some extent, reflect the dynamics of population succession in urban areas. This corresponds to case $\mathbf{B}$ in Fig. 3. Consider the payoff matrix in Fig. $3 \mathbf{B}$, and imagine that an individual of group 1 happens to be located in the neighborhood of group 2. For the previous residents, this may not change a lot. However, it attracts other members of group 1, who are not yet living in a neighborhood of group 1 individuals. This can trigger collective migration of other group 1 members. Since interactions between members of group 1 and 2 bring positive payoffs only for members of group 1, group 2 will finally leave for new places. Of course, this process repeats, just like the circle of population succession in Sec. 1.2.

One may notice that, here, we do not differentiate the intention to migrate and the actual migratory behavior. In daily life, however, the movement of people is restricted by much more factors such as wealth and time, so people do not migrate often, even if they are motivated to move. But in our simulations as well, migration activity after a few iterations is small: Starting with high migration rate, due to the artificial choice of a random initial distribution, the migration rate quickly drops to a low level [12]. Of course, other factors determining migration can be easily added to the above proposed framework of migration games.

\subsection{Promotion of Cooperation in the Prisoner's Dilemma}

The prisoner's dilemma is an important paradigm for studying the emergence of cooperation among selfish individuals. Nevertheless, studies of the prisoner's dilemma on lattices have not fully explored the effect of mobility. It would be logical, of course, for people to evade areas with a high level of defection, and to settle down in areas dominated by cooperators. But would cooperative areas be able to survive invasion attempts by defectors or even to spread in space? To answer these questions, we will now focus on the effects of success-driven migration on the spatial population structure and the level of cooperation.

Fig. 4 compares the migration-only case with $\mathrm{M}=5$ (left) with the imitation only case corresponding to $\mathrm{M}=0$ (center) and the combined imitation-and-migration case with $\mathrm{M}=5$ (right). In the imitation-only case, the proportion of cooperators is greatly reduced. However, the combination of migration and imitation strikingly promotes the level of cooperation. Our explanation is that, when individuals have mobility, cooperative clusters are more likely to be promoted in the presence of invasion 
attempts of defectors. We can see that, in the migration-only case, cooperators manage to aggregate and to form clusters. Although defectors attempt to enter cooperative clusters, they finally end up at the boundaries of cooperative clusters, as cooperators split to evade defectors and re-aggregate in new places, where defectors are excluded. In the prisoner's dilemma, it is guaranteed that $2 R>T+S$, which means that the "attractive force" between cooperators is mutual and strong, while the interaction between a cooperator and a defector leads to a unilateral attractive force $(T>0)$. When $S<0$, a cooperator replies to defectors even in a repulsive way. Therefore, defectors are less successful in joining or entering cooperative clusters than cooperators are.

Configurational analysis [12] (see Fig. 5), indicates that, when $P=$ $S=0$, and an individual only interacts with $k=4$ nearest neighbors, a cooperative cluster can turn a defector into cooperator by unconditional imitation for $T<1.5 R$, when the defector is surrounded with one or two cooperators. When a defector is surrounded with three cooperators, cooperators can resist the invasion of a defector, even if the temptation value $T$ is as high as $\frac{4}{3} R$. A defector can invade one of the nearby cooperators, if its neighborhood is fully occupied by cooperators. Therefore, the formation of compact clusters is important to support the spreading of cooperation. If $k>4$, the spreading of cooperation occurs even for higher value of $T$.

In spatial games without mobility, the occurrence of a compact cooperative clusters mainly depends on the initial distribution. Giving mobilities, the migratory behavior can significantly accelerate the formation of compact clusters, and promote the level of cooperation.

Quantitative studies of how migration can promote the level of cooperation have to compare the fraction of cooperators in situations with mobility and without. Fig. 6 shows the amplification factor, defined as

$$
\delta(T, M)=\frac{f_{M}^{T}}{f_{0}^{T}}
$$

$f_{M}^{T}$ is the fraction of cooperators, given the mobility range $M$ and a temptation value of $T$. We can see that the level of cooperation in the prisoner's dilemma is promoted in a large parameter area, if the punishment $P$ and the sucker's payoff are roughly comparable in size.

However, we have not yet studied the robustness of the migration mechanism so far. One may imagine that, once a defector would manage to invade a cooperative cluster, it may turn neighboring cooperators into defectors as well, thereby eliminating cooperation eventually. While in 


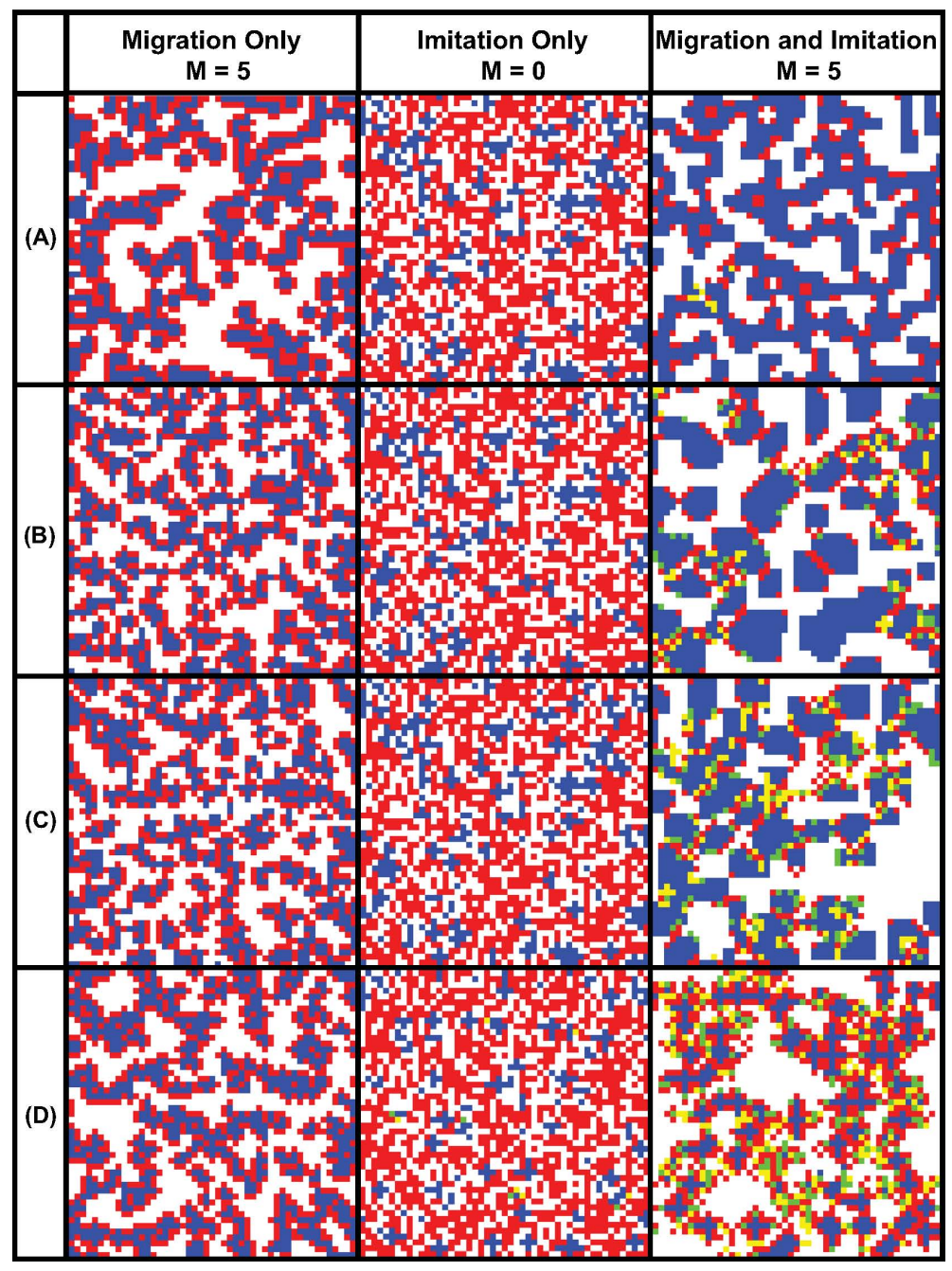

Fig. 4. Simulation results for $49 \times 49$ grids with a density of 0.6 for the migration-only case (left), the imitation-only case of conventional spatial games (middle) and the combination of imitation with migration (right). The color code is chosen as follows: blue $=$ player of group 1 , red $=$ player of group 2, yellow = player of group 1 who turned into a player of group 2 in the last iteration, green = player of group 2 who turned into a player of group 1 in the last iteration, white $=$ empty site. (A) $P_{11}=R=1, P_{12}=S=-0.2, P_{21}=T=1.4, P_{22}=P=0$. The payoff matrix corresponds to a prisoner's dilemma. (B) $P_{11}=R=1$, $P_{12}=S=0, P_{21}=T=1.4, P_{22}=P=0$. The sucker's payoff is set to zero to be compatible with the payoff matrix studied by Nowak and May. (C) $P_{11}=R=1, P_{12}=S=-0.2, P_{21}=T=1.4, P_{22}=P=0$. The migration and imitation step are inverted here, i.e. an individual first imitates, then migrates. (D) $P_{11}=0.59, P_{12}=0.18, P_{21}=1, P_{22}=0$. In the snow-drift game, similar structures are found in the migration-only and imitation-only case. Giving the possibility to move, frequent switches of strategies are observed. See main text for details. 

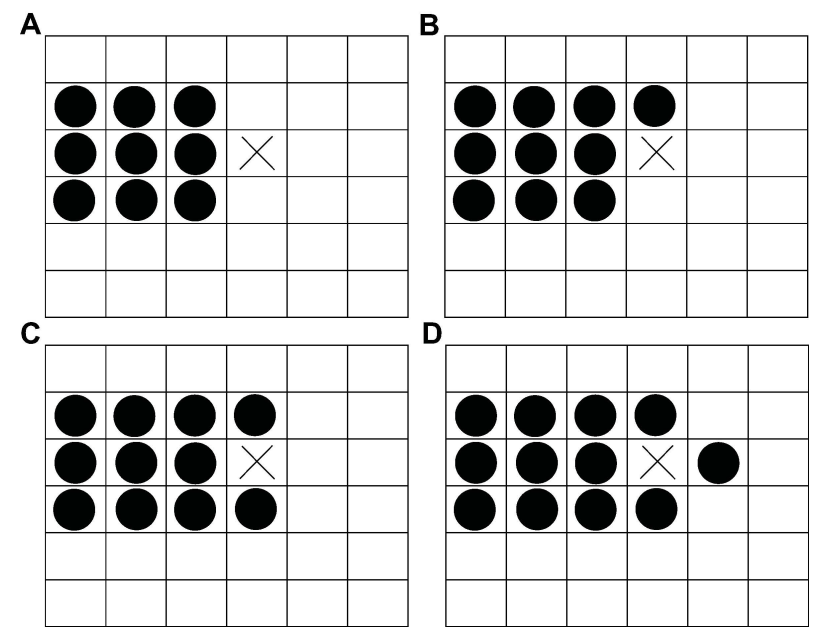

Fig. 5. Analysis of the invasion of a cluster of cooperators (black circles) by a single defector (cross). For the simplicity of analysis, we assume $S=$ $P=0$ here, but one can easily generalize the analysis to the situations with $P>S>0$. (A) Scenario 1: The payoff for the defector is $T$, while its neighboring cooperator obtains a payoff of $3 R$. Since $3 R>T$, the defector will become a cooperator. (B) Scenario 2: The payoff for the defector is $2 T$, while the maximal payoff among neighboring cooperators is $3 R$. In order to make the defector become a cooperator, we need $3 R>2 T$, i.e. $T<1.5 R$. (C) Scenario 3: The defector obtains 3T, while the maximal payoff of neighboring cooperators is $3 R$. In order to turn the defector into a cooperator, the inequality $3 R>3 T$ must be satisfied, i.e. $T<R$. This condition can never be met in the prisoner's dilemma. In order that cooperators do not copy the defector, $4 R>3 T$ must be satisfied, i.e. $T<\frac{4}{3} R$. (D) Scenario 4: The payoff for the defector is $4 T$, the maximal payoff of cooperators in the whole community is $4 R$. Because of $T>$ $R$, the defector can invade the cooperators nearby. Once a cooperator becomes a defector however, the payoff for the defectors will be reduced from $4 T$ to $3 T$, which may stop the further invasion of defectors 


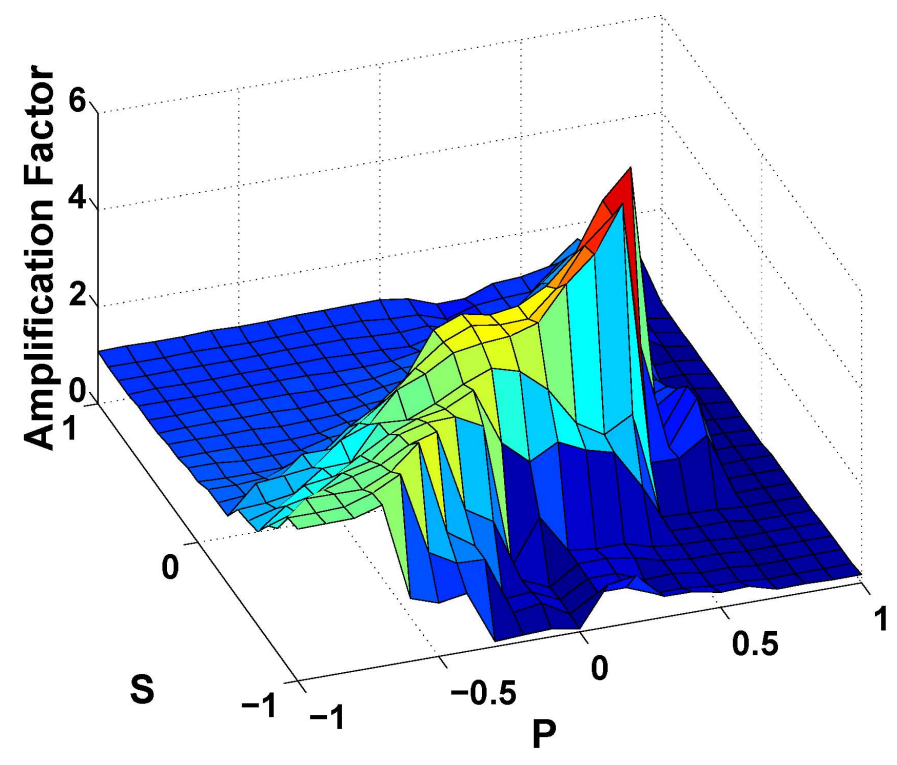

Fig. 6. Amplification factor of the level of cooperation by migration $(M=$ 5 ) as a function of the sucker's payoff $P_{12}=S$ and the punishment $P_{22}=$ $P$ in the prisoner's dilemma $(S<P)$ and the snow drift game $(S>P)$. The simulation was performed for $99 \times 99$ grids with a density of 0.6 . $P$ and $S$ were varied between -1 and 1 , the payoffs $R=1$ and $T=1.3$ were left fix. 
a noiseless environment, a defector cannot enter the center of a compact cooperative cluster, "noise" could make it happen with a certain probability. As defectors in a cooperative cluster can spread (see Fig. 5D), noise could therefore be thought to destroy the enhancement of cooperation by success-driven migration. Very surprisingly, this is not the case!

In order to verify that success-driven migration robustly promotes cooperation, we have implemented 3 kinds of noises. In each time step, a certain proportion $y$ of individuals was selected to perform the following operations after the respective migration and imitation steps:

Noise 1: The selected players' locations were exchanged with a randomly chosen neighboring site ("neighborhood flipping").

Noise 2: The selected players' strategies were flipped, i.e. cooperation was replaced by defection and vice versa ("strategy flipping").

Noise 3: The selected players were removed from the grid, and an equal number of players was created at randomly chosen free sites, in order to mimic birth and death processes. The newly born players had a $50 \%$ chance to be cooperators and a $50 \%$ chance to be defectors.

Fig. 7 shows the time evolution of the number of cooperators with noise strength $y=2 \%$ and $y=10 \%$ respectively. Without mobility, noise reduces the number of cooperators greatly. For a mobility range $M=5$, however, we surprisingly find that the level of cooperation can be still maintained at a high level. With $2 \%$ noise, noises 1 and 3 can even increase the number of cooperators compared to the no-noise case!

In order to understand how noise can promote cooperation to a level higher than the level in a noiseless system, we may define a kind of potential energy function of the system by the negative total payoff:

$$
E=-T^{\prime}
$$

In a noiseless system, each individual's migration step will increase $T$, and reduce the potential energy of the system, [see Eq. (11)]. Then an individual will adopt the most successful strategy within its neighborhood. The imitation step and noise can flip the strategies and increase the energy of the system. Just as for the energy functions in spin glass models [42], recurrent neural networks [43] and Boltzman machines [44, there are many local minima of $E$ in the migration game, one of which is reached within a few iteration steps. Then the noiseless system behaves stationary. It is stuck in the corresponding meta-stable state, which is highly dependent on the initial condition. When noise occurs, it can perturb the meta-stable state of the system and drive it to more stable states (see Fig. 8). Only if the noise level is too strong, the system behaves pretty 


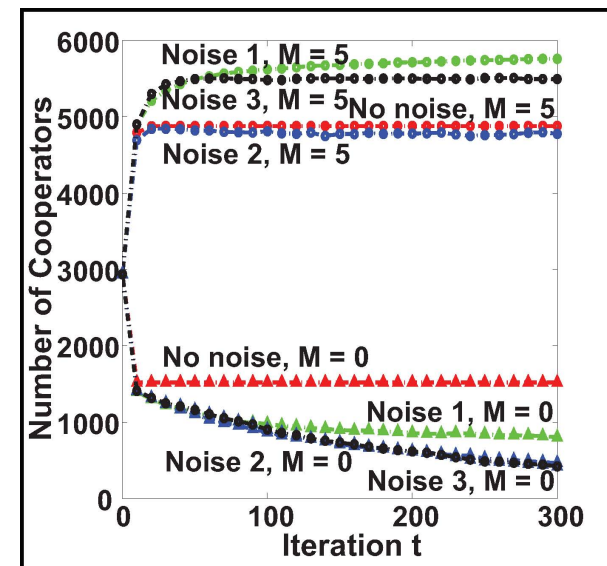

(A) $2 \%$ noise

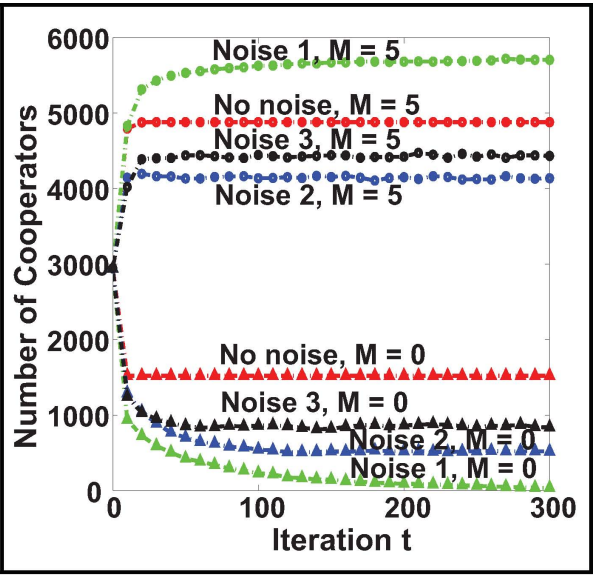

(B) $10 \%$ noise

Fig. 7. Time evolution of the number of cooperators. Here, the simulation is performed on $99 \times 99$ grids with a density of 0.6 and payoffs $P_{11}=R=1$, $P_{12}=S=0, P_{21}=T=1.3$, and $P_{22}=P=0$.

much in a random way. Therefore, in conclusion, moderate levels of noise do not destroy a high level of cooperation - they can even support it.

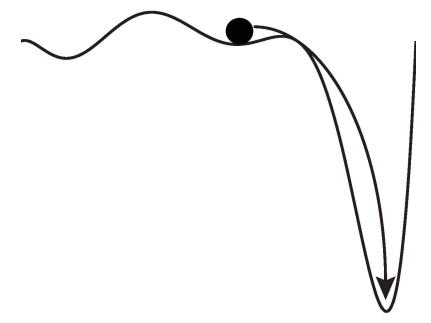

Fig. 8. Noise can make the system leave a sub-optimal state and reach the globally optimal state.

Our results for the conventional prisoner's dilemma with $P>S$ confirm the robustness of migration as a mechanism to promote cooperation (see Fig. 9). One can easily see that, without mobility, the proportion of cooperators is close to zero, while there is still a certain small number of 
cooperators in the environment without noise. However, when individuals can move, cooperation is significantly increased due to the spontaneous formation of clusters. Imagine that a defector is located in the center of a cooperators' cluster. In the beginning, defection can invade cooperation due to the higher payoff (see Fig. 5D). But once a neighboring cooperator becomes a defector, a defector's payoff is reduced from $4 T$ to $3 T$, if $P=0$. If more cooperators turn into defectors, the payoff will be further reduced. Therefore, the exploitation of cooperators by defectors is self-inhibitory, if individuals copy better performing neighbors. Furthermore, a splitting of a cooperative cluster can occur, since cooperators try to move to more favorable neighborhood as soon as defectors are approaching them. The migration of those cooperators can encourage other cooperators, whose payoff depends on the mutual interactions, to move as well. That is, defectors may trigger a collective movement of cooperators. Of course, the newly formed cooperative clusters are also likely to be invaded by neighborhood or strategy flipping, or birth and death process. However, this will just repeat the above mentioned migration process. Therefore, cooperators can survive and spread even in a noisy world. Such a dynamical change of the population structure through invasion and succession reflects various features of the migratory behavior observed in reality (see sec. 1.2).

In the migration rule studied above, we assume that a favorable neighborhood can be determined by fictitious play, i.e. some low-cost interactions with the people in that neighborhood ("neighborhood testing"). One may think that this is difficult in reality as it requires to reveal people's strategies. However, one may also argue that people tend to migrate to high-quality residential areas, which provide better education for children, a low crime rate, and other social welfare. In fact, neighborhoods are often "labeled", and it may be assumed that this label (the appearance and character of a neighborhood) depends on the total cumulative payoffs (the accumulated wealth) of the residents in the neighborhood. Therefore, one could assume that individuals try to move to the neighborhood with the highest cumulative payoff. The success-driven migration based on such a wealth-based "neighborhood tagging" is examined in Fig. 10. Again, we find that migration promotes the formation of cooperative clusters and an enhanced level of cooperation. 


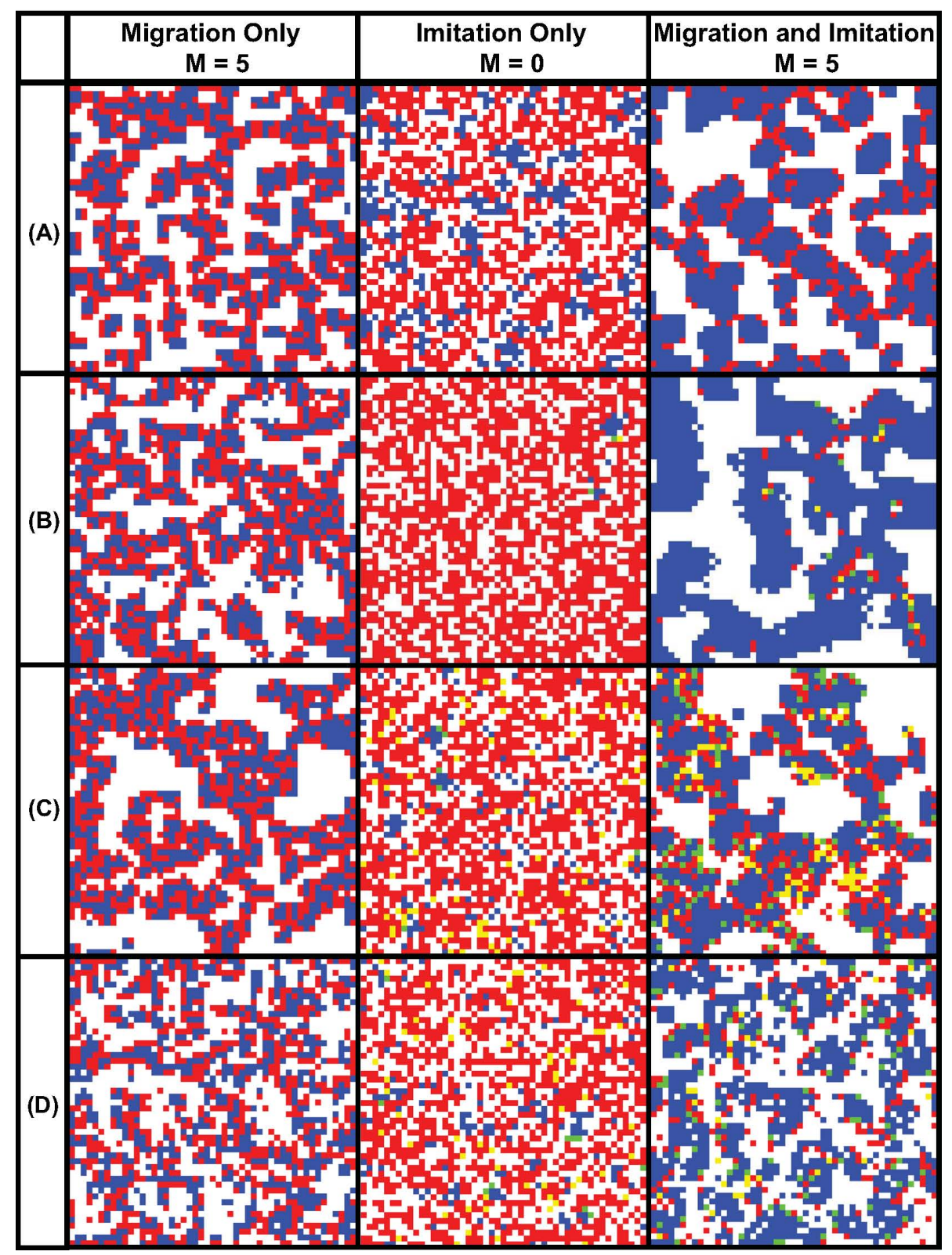

Fig. 9. Effect of different kinds of noises on the outcome of the spatial prisoner's dilemma with migration but no imitation (left), with imitation, but no migration (center), and with both, migration and imitation (right). The simulation is performed on $49 \times 49$ grids with a density of 0.6 . The color code is chosen as follows: red $=$ defector, blue $=$ cooperator, green $=$ defector who became a cooperator, yellow $=$ cooperator who turned into a defector in the last iteration, white = empty site. While the resulting level of cooperation is very small in the conventional imitation-only case (center), the additional consideration of migration results in large levels of cooperation even in the presence of different kinds of noise. (A) No noise. (B) Noise 1 (neighborhood flipping). (C) Noise 2 (strategy flipping). (D) Noise 3 (birth and death). The payoffs were $T=1.3, R=1, P=0.1$, and $S=0$ in all cases. See main text for details. 


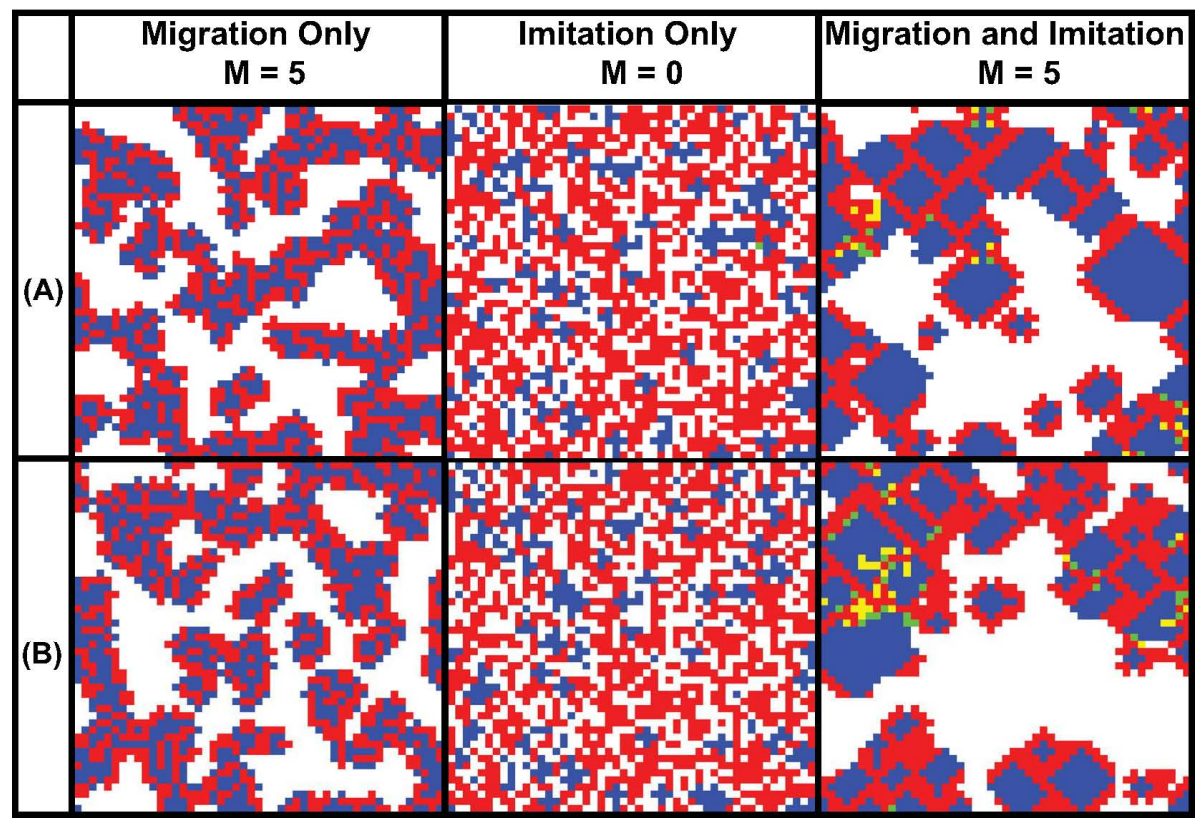

Fig. 10. Migratory prisoner's dilemma with wealth-based neighborhoodtagging rather than neighborhood testing as before. The simulation is performed on $49 \times 49$ grids with a density of 0.6 . The color code is chosen as follows: red $=$ defector, blue $=$ cooperator, green $=$ defector who became a cooperator, yellow $=$ cooperator who turned into a defector in the last iteration, white $=$ empty site. (A) $P_{11}=R=1, P_{12}=S=0, P_{21}=$ $T=1.4, P_{22}=P=0$. (B) $P_{11}=R=1, P_{12}=S=0, P_{21}=T=1.4$, $P_{22}=P=0$, as in (A), but the update rule is inverted, i.e. an individual, first imitates, then migrates. In both cases, one can see that, without mobility, the proportion of cooperators becomes very low. However, when success-driven migration is possible, cooperation can spread, even if the sequence of the migration step and the unconditional imitation step is inverted. 


\section{Conclusions}

We have introduced the concept of migration games by considering successdriven motion. Migration games can easily reproduce macroscopic stylized facts of various social phenomena based on individual actions and interactions. Typical examples are population succession and residential segregation. These aggregate outcomes emerge from the interactions between individuals in a non-trivial way, and a theoretical analysis allows one to qualitatively understand the relation between the microscopic interactions and the emerging macroscopic phenomena. Nevertheless, further studies are required to fully elaborate the micro-macro link in a quantitative way.

For the prisoner's dilemma, we have shown that self-organized cooperative structures can promote the level of cooperation. Moreover, we have verified that the enhancement of cooperation by success-driven motion is robust to different kinds of noise. Surprisingly, we even find that moderate noise levels can promote the cooperation level further. The underlying mechanism is that, in the migration game, success-driven motion will monotonously increase the total payoff in the noiseless system, which however can lead the system into a locally optimal state. The effect of noise can drive the system out of local optima towards the globally optimal state.

The framework of migration games is quite flexible and allows the integration of other interactions to study further social processes.

The spatial structure in our simulation is very simple, and the neighborhood depends only on an individual's position. Real cities are more complex and show a co-evolutionary dynamics. For example, the city structure can reflect the distribution of social status groups. Early models like the Burgess concentric zone model [45] divides the city into specific areas separated by status rings. The city center is located in the middle of the circle, around which newer, higher-quality housing stocks tend to emerge at the perimeter of the city. Therefore, the growth of the city center will expand adjacent residential zones outwards.

Status segregation is quite obvious in such an idealized model of city structure. Poor people or new immigrants may only afford low quality housing. Middle class people live in less compacted neighborhoods. Rich people tend to accumulate in particular quarters.

Burgess's model is based on the bid rent curve. Recognizing that some poor people prefer to live near the main transportation arteries and com-

mercial establishments, Hoyt [46] modified the concentric zone model to take this into account. In Hoyt's model, cities tend to grow in wedge- 
shaped patterns or sectors. Major transportation routes are emanating from the central business district (CBD). The residential areas for lower income people are located adjacent to the industrial quarters, while upper class neighborhoods are far away from industrial pollution and noise.

It would be natural to extend migration games in order to study the co-evolutionary dynamics of population structure and urban growth. On the long run, we hope this will contribute to a better understanding and planning of the population dynamics in a city.

Further research work can also study conflicts related with migratory behavior, as has been revealed by the empirical research [4748].

\section{References}

1. Clark, W. A. and Fossett, M. Understanding the social context of the Schelling segregation model. Proc. Natl. Acad. Sci. 105, 4109 - 4114 (2008).

2. Fossett, M. Ethnic preferences, social distance dynamics, and residential segregation: theoretical explorations using simulation analysis. Journal of Mathematical Sociology 30, 185-274 (2006).

3. Duncan, O. D. and Duncan, B. Residential distribution and occupational stratefication. American Journal of Sociology 60, 493-503 (1955).

4. Reardon, S. F. and Firebaugh, G. Response: Segregation and social distance a generalized approach to segregation measurement. Sociological Methodology 32, 85-101 (2002).

5. Schelling, T. C. Dynamic models of segregation. J. Math. Socio. 1, 143-186 (1971).

6. Schelling, T. C. Micromotives and Macrobehavior (W. W. Norton, New York, 1978).

7. Massey, D. S. American apartheid: segregation and the making of the underclass. American Journal of Sociology 96, 329-357 (1990).

8. Yinger, J. Closed Doors, Opportunities Lost: The Continuing Costs of Housing Discrimination (Russell Sage Found, New York, 1995).

9. Macy, M., Rijt, A. V. D. Ethnic preferences and residential segregation: Theoretical explorations beyond Detroit. Journal of Mathematical Sociology 30, 275-288 (2006).

10. Helbing, D. and Platkowski T. Drift- or fluctuation-induced ordering and selforganization in driven many-particle systems. Europhys. Lett 60, 227-233 (2002).

11. Helbing, D. and Vicsek, T. Optimal self-organization. New Journal of Physics 1, 13.1-13.17 (1999).

12. Helbing, D. and $\mathrm{Yu}, \mathrm{W}$. Migration as a mechanism to promote cooperation. Advances in Complex Systems 11, 641-652 (2008).

13. Cressey, P. F. Population succession in Chicago: 1898-1930. American Journal of Sociology 44, 59-69 (1938).

14. Nowak, M. A. Five rules for the evolution of cooperation. Science 314, 1560 (2006).

15. Axelrod, A. The Evolution of Cooperation (Basic Books, New York, 1984).

16. Griffin, A. S., West, S. A. and Buckling, A. Cooperation and competition in pathogenic bacteria. Nature 430, 1024-1027 (2004).

17. Von Neumann, J. and Morgenstern, O. The Theory of Games and Economic Behavior (Princeton University Press, Princeton, 1944).

18. Fudenberg, D. and Tirole, J. Game Theory (MIT Press, Cambridge, 1991). 
19. Diekmann, A. Volunteer's dilemma. Journal of Conflict Resolution 29, 605-610 (1985).

20. Flache, A. and Hegselmann, R. Do irregular grids make a difference? Relaxing the spatial regularity assumption in cellular models of social dynamics. In: Journal of Artificial Societies and Social Simulation 4, no. 6 (2001), see http : //www.soc.surrey.ac.uk/JASSS/4/4/6.html.

21. Schuster, P. and Sigmund, K. Replicator dynamics. J. Theor. Biol. 100, 533-538 (1983).

22. Nowak, M. A. and May, R. M. Evolutionary games and spatial chaos. Nature 359, 826-829 (1992).

23. Hauert, C. and Doebell, M. Spatial structure often inhibits the evolution of cooperation in the snowdrift game. Nature 428, 643-646 (2004).

24. Park, R. E. Human ecology. American Journal of Sociology 42, 1-15 (1936).

25. Schwirian, K. P. Models of neighborhood change. Ann. Rev. Sociol. 9, 83-102 (1983).

26. Weidlich, W. Sociodynamics. A Systematicc Approach to Mathematical Modeling in the Social Sciences (Harwoord Academic, Amsterdam, 2000).

27. Granovetter, M. Threshold models of collective behavior. American Journal of Sociology 83, 1420-1443 (1978).

28. Harris, D. R. "Property values drop when blacks move in, because...": Racial and socioeconomic determinants of neighborhood desirability, American Sociological Review 64, 461-479 (1999).

29. Hoogendoorn, S. and Bovy, P. H. L. Simulation of pedestrian flows by optimal control and differential games. Opim. Control Appl. Mech. 24, 153-172 (2003).

30. Vainstein, M. H. and Arenzon, J. J. Disordered environments in spatial games. Phys. Rev. E 64, 051905 (2001).

31. Vainstein, M. H., Silva, A. T. C. and Arenzon, J. J. Does mobility decrease cooperation? J. Theor. Biol. 244, 722-728 (2006).

32. Sicardi, E. A., Fort, H., Vainstein, M. H. and Arenzon, J. J. Random mobility and spatial structure often enhance cooperation. J. Theor. Biol. 256, 240-246 (2009).

33. Epstein, J. M. Generative Social Science (Princeton University Press, Prienceton, 2006).

34. Young, H. P. Individual Strategy and Social Structure (Princeton University Press, Princeton, 1998).

35. Macy, M. W. Learning to cooperate: stochastic and tacit collusion in social exchange. American Journal of Sociology 97, 808-843 (1991).

36. Macy, M. W. and Flache, A. Learning dynamics in social dilemmas. Proc. Natl. Acad. Sci. 99, 7229-7236 (2002).

37. Wolfram, S. Theory and Applications of Cellular Automata (World Scientific Publication, Singapore, 1986).

38. Chopard, B. and Droz, M. Cellular Automata Modeling of Physical Systems (Cambridge University Press, Cambridge, 1998).

39. Berlekamp, E. R., Conway, J. H. and Guy, R. K. Winning Ways for Your Mathematical Plays (Academic Press, New York, 1982).

40. Huberman, B. A. and Glance, N. S. Evolutionary games and computer simulations. Proc. Natl. Acad. Sci. 90, 7716-7718 (1993).

41. Helbing, D., Farkas, I. and Vicsek, T. Simulating dynamical features of escape panic. Nature 407, 487-490 (2000).

42. Kirkpatrick, S., Sherrington, D. Infinite-ranged models of spin-glasses. Physical Review B 17, 4384-4403 (1978). 
43. Hopfield, J. J. Neural networks and physical systems with emergent collective computational abilities. Proc. Natl. Acad. Sci. 79, 2554-2558 (1982).

44. Ackley, D. H., Hinton, G. E., Sejnowski, T. J. A learning algorithm for Boltzmann machines. Cognitive Science 9, 147-169 (1985).

45. Burgess, E. W. Residential segregation in American cities. Annals of the American Academy of Political and Social Science 140, 105-115 (1928).

46. Hoyt, H. The Structure and Growth of Residential Neighborhoods in American Cities Washington (Federal Housing Administration, 1939).

47. Lim, M., Metzler, R. and Yam, Y. B. Global pattern formation and ethnic/cultural violence. Science 317, 1540-1544 (2007).

48. Weidmann, N. B., and Kuse, D. WarViews: Visualizing and animating geographic data on civil war. Accepted for publication in International Studies Perspectives. 